\title{
Critical path analysis for continuum percolation ${ }^{\text {th }}$
}

\author{
Jiř́ Černý \\ Weierstrass Institute for Applied Analysis and Stochastics, Mohrenstr. 39, 10117 Berlin, Germany \\ Received 21 November 2002; accepted 19 May 2003 \\ Available online 11 September 2004
}

\begin{abstract}
We prove the validity of the Critical path analysis for a continuum percolation model close to Golden-Kozlov one. This is obtained in the limit of strong disorder.
\end{abstract}

(C) 2004 Elsevier SAS. All rights reserved.

\section{Résumé}

On montre la validité de "Critical path analysis" pour un modèle de percolation continue proche de celui de Golden-Kozlov. Le résultat est obtenu à la limite de grand désordre.

(c) 2004 Elsevier SAS. All rights reserved.

Keywords: Conductivity; Continuum percolation; Highly disordered systems; Homogenisation

\section{Introduction}

One of the central issues of the theory of disordered materials is the determination of effective properties (like electrical conductivity or fluid permeability) from the knowledge of the micro-structural properties. In many areas of practical importance, the probability distribution of local physical characteristics is very broad. An interesting property of these so-called "highly disordered" systems is that the effective conductivity of the sample can often be approximated by the conductivity of a very small part of it. Such part is usually composed by a small number of paths that contribute overwhelmingly to the effective conductivity. It is thus important to find out the conditions that lead to this behaviour, since it is usually far less complex to compute the conductivity of a small number of paths than of the whole sample.

\footnotetext{
This work was supported by grant FNRS 21-54118.98.

E-mail address: cerny@ wias-berlin.de (J. Černý).
} 
This idea was, for the first time, introduced by [1] and is known in the physical literature as "Critical Path Analysis (CPA)". It was used successfully in many areas of physics $[2,8]$. However, rigorous investigations are sparse up to now [4,11].

It should be obvious that the creation of strongly conducting paths (and thus the calculation of effective properties of the sample) is connected with the percolation of highly conducting areas. Let us explain this relation heuristically on a simple model. The procedure of reduction of the sample to a small set of "critical paths" follows [3]. We will call this procedure a "pruning procedure".

Let $\Lambda_{N}$ be the box of size $N$ in $\mathbb{Z}^{2}$ and let $\mathbb{L}_{N}$ be the set of all bonds connecting nearest neighbours in $\Lambda_{N}$. Assign to each bond $b \in \mathbb{L}_{N}$ a random i.i.d. conductivity $c_{b}$. We want to compute the conductivity of the sample with the potential difference applied on the left and right edge of the box.

Now we start describing the "pruning procedure". First, we sort all the bonds in the graph $\left(\Lambda_{N}, \mathbb{L}_{N}\right)$ according to their conductivity. Then we delete all the bonds from the graph except the bonds that are contained in left or right edge of the box, and we start to re-add them bond by bond in the order of decreasing conductivity. After each step we check for loops. If there is a loop, we delete the bond just added and we continue with the next one. At the beginning of this procedure, there will be no connection between the left and right edge. After sufficiently many steps, adding the next bond produces a connection between the left and right edge. We stop the procedure at this moment. What we get at this point is a treelike structure containing one connection from left to right and many dead-ends that we can delete safely, because they do not contribute to the transport. The conductivity of this connection is easy to obtain. If the distribution of local characteristics is broad enough, then the CPA claims that the conductivity of this connection is close to the conductivity of the graph before the pruning.

One can go further in this type of reasoning. The conductivity of one-dimensional path of conducting elements with conductivities drawn from a very broad distribution is essentially determined by the element with the smallest conductivity. Applying this to the path constructed by pruning, one can conclude that the conductivity of the box is not far from the conductivity of the bond we have added as the last one. If $N$ is large enough, the conductivity of the last added bond should be close to the largest value of conductivity $c^{\star}$ such that the bonds with $c_{b}>c^{\star}$ percolate. More precisely, denoting by $F(x)=\mathbb{P}\left(c_{b} \leqslant x\right)$ the distribution function of the local conductivity and by $p_{c}$ the percolation threshold of the bond percolation, the conductivity of the box should be close to

$$
c^{\star}=\sup \left\{x: 1-F(x) \geqslant p_{c}\right\} .
$$

In this paper we construct a model where the above heuristic can be proved. The effective conductivity will be very close (at least in the limit of strong disorder) to the "critical local conductivity". This can be interpreted as a justification of the CPA for this model. The model we use is a continuous generalisation of the "chess-board" model used in [4].

\section{Definitions and results}

We consider the following two-dimensional medium. Let $X=X(\omega), \omega \in \Omega$ be a homogeneous Poisson point process with density $\lambda$ defined on some probability space $\Omega$ (see Section 3 for the definition). For every point $x \in \mathbb{R}^{2}$ let $S(x)=S(x, \omega)$ denote the minimal distance to some point of $X$,

$$
S(x)=\inf \{d(x, y): y \in X\},
$$

where $d(\cdot, \cdot)$ denotes the Euclidean distance of two points. We define the local conductivity of the medium by

$$
\sigma(x, \mu)=\sigma(x, \mu, \omega)=\exp (\mu S(x)),
$$

where $\mu$ is a positive parameter. That means that our medium can be considered as the set of insulating grains with the centres in the points of the point process. The parameter $\mu$ controls the amount of disorder of the system. We will be interested in the case where $\mu$ is very large. 
The medium we have just defined is obviously statistically isotropic. Thus, its macroscopic properties can be described by one scalar effective conductivity $\sigma^{\star}(\mu, \omega)$ defined as follows. Let $\Lambda_{N}$ be the box $[0, N]^{2}$ and let $u_{N}(x, \mu)=u_{N}(x, \mu, \omega)$ be the solution of the system

$$
\begin{aligned}
& \operatorname{div}\left(\sigma(x, \mu) \nabla u_{N}(x, \mu)\right)=0, \quad x=\left(x^{1}, x^{2}\right) \in \Lambda_{N}, \\
& u_{N}(x, \mu)=0, \quad x^{1}=0, \\
& u_{N}(x, \mu)=N, \quad x^{1}=N, \\
& \frac{\partial u_{N}(x, \mu)}{\partial x^{2}}=0, \quad x^{2} \in\{0, N\} .
\end{aligned}
$$

The function $u_{N}(x, \mu)$ is the electrical potential in the box $\Lambda_{N}$ with the prescribed boundary conditions. Let $J_{N}(\mu)=J_{N}(\mu, \omega)$ denote the overall flow through the vertical line $x^{1}=b, b \in(0, N)$,

$$
J_{N}(\mu)=\int_{0}^{N} \sigma\left(\left(b, x^{2}\right), \mu\right) \frac{\partial u_{N}\left(\left(b, x^{2}\right), \mu\right)}{\partial x^{1}} \mathrm{~d} x^{2},
$$

which obviously does not depend on $b$. The effective conductivity is then defined by

$$
\sigma^{\star}(\lambda, \mu, \omega)=\lim _{N \rightarrow \infty} \frac{1}{N} J_{N}(\mu, \omega) .
$$

Since our medium is evidently ergodic, it follows from the results of homogenisation theory that this limit exists almost surely and does not depend on $\omega$ (see [7, Theorem 7.4]).

To state our first theorem we need one quantity from the continuum percolation (for a good survey see [10]). It is well known that there exists a nontrivial value $S_{c}(\lambda)$, such that the set $\left\{x \in \mathbb{R}^{2}: S(x) \leqslant r\right\}$ percolates iff $r>S_{c}(\lambda)$, and its complement percolates iff $r<S_{c}(\lambda)$. We call $S_{c}(\lambda)$ the critical radius. As we have noted in the introduction, this value should be important for the estimation of the effective conductivity in the limit of the strong disorder. Actually, we have

Theorem 2.1. For almost all realisations of the medium the value of the effective conductivity depends only on the parameters $\lambda$ and $\mu$ and asymptotically satisfies

$$
\lim _{\mu \rightarrow \infty} \frac{1}{\mu} \log \sigma^{\star}(\lambda, \mu)=S_{c}(\lambda) .
$$

To clarify the relation of this result with Eq. (1) observe that Theorem 2.1 roughly says that $\sigma^{\star}(\lambda, \mu) \sim$ $\exp \left(\mu S_{c}(\lambda)\right)$. This value is the largest $\sigma$ such that the domain where the conductivity is larger or equal to $\sigma$ percolates.

The next theorem shows something that resembles the pruning that was described before, and also clarifies the meaning of Theorem 2.1. The pruning in this case cannot be defined in the same way as for the square lattice. However, it is possible to reduce our medium and to obtain a medium that essentially consists of points connected by tubes. These points will not be located on the square lattice, but this does not pose major problems for the pruning procedure.

As we have already noted, our medium can be regarded as an ensemble of insulating grains in the plane. Between every pair of neighbouring grains there is a domain where the conductivity is large. The structure of these grains can be identified with the Voronoi tessellation defined by the process $X(\omega)$. If $\mu$ is large, the conductivity decreases very rapidly with the distance from the borders of Voronoi cells. Hence, the contribution of a small neighbourhood of these borders to the effective conductivity should be very important. Thus, we should not make a large error if we consider the rest of the medium as totally insulating. We get a medium that consists only of the thin tubes around the borders of the Voronoi cells. 
More precisely, let $\mathcal{V}(\omega) \subset \mathbb{R}^{2}$ denote the set of borders of Voronoi cells around the points of $X(\omega)$ and let $\rho>0$ be a small positive constant. We define first the modified conductivity $\tilde{\sigma}(x)$

$$
\tilde{\sigma}_{\rho}(x, \mu)= \begin{cases}\sigma(x, \mu) & \text { if } d(x, \mathcal{V})<\rho \\ 0 & \text { if } d(x, \mathcal{V})>2 \rho .\end{cases}
$$

In the domain between $\rho$ and $2 \rho$ the function $\tilde{\sigma}_{\rho}(x)$ continuously and "monotonically" interpolates between the values on the boundary of this domain. The way how the interpolation is done is not important. We use it only to make the conductivity continuous and to avoid problems with the boundary conditions on the walls of the tubes.

The medium $\tilde{\sigma}_{\rho}(x)$ can be "pruned" further. It is obvious that at each bond $b$ of $\mathcal{V}$ there is exactly one point $s_{b}$ where the function $S(x)$, and thus also $\tilde{\sigma}_{\rho}(x, \mu)$, has a saddle point. The flow passing through the tube around the bond $b$ should therefore go through an area where the local conductivity is close to $\sigma\left(s_{b}, \mu\right)$. Intuitively, the conductivity of the whole tube around $b$ should be proportional to this value, because far from the saddle point the local conductivity is much larger. Actually, it can be easily proved at least for $\mu$ large enough, but we will not need this claim later. Using this observation, one sees that the bonds with $\sigma\left(s_{b}, \mu\right)$ very small should not contribute too much to the overall conductivity. So we delete them. More formally, let $\mathcal{V}_{\delta}$ be the subset of $\mathcal{V}$ containing only the bonds with $S\left(s_{b}\right)>S_{c}(\lambda)-\delta$, i.e. the bonds that are far from the points of $X$. Let us define another modified medium $\hat{\sigma}_{\rho, \delta}(x, \mu)$ in the same way as we defined $\tilde{\sigma}_{\rho}(x, \mu)$ but using $\mathcal{V}_{\delta}$ instead of $\mathcal{V}$ :

$$
\tilde{\sigma}_{\rho, \delta}(x, \mu)= \begin{cases}\sigma(x, \mu) & \text { if } d\left(x, \mathcal{V}_{\delta}\right)<\rho, \\ 0 & \text { if } d\left(x, \mathcal{V}_{\delta}\right)>2 \rho .\end{cases}
$$

The medium $\hat{\sigma}_{\rho, \delta}$ consists of the tubes from $\tilde{\sigma}_{\rho}$ with large conductivity.

Note, that we do not define pruning in the inductive way that we have described before. The "pruned" medium $\hat{\sigma}_{\rho, \sigma}(x)$ does not consist of a single one-dimensional path crossing the box and it contains more tubes than it should. However, if the parameter $\delta$ is small (how small it should be, depends on the size of the box that we consider) the difference should not be substantial.

We use $\tilde{\sigma}_{\rho}^{\star}(\lambda, \mu)$ and $\hat{\sigma}_{\rho, \delta}^{\star}(\lambda, \mu)$ to denote the effective conductivities of the modified media. Then we have:

Theorem 2.2. For every $\delta>0$ and $\rho>0$, the effective conductivities of the pruned media $\tilde{\sigma}_{\rho}^{\star}(\lambda, \mu)$ and $\hat{\sigma}_{\rho, \delta}^{\star}(\lambda, \mu)$ satisfy the same relation as the original medium, i.e.

$$
\lim _{\mu \rightarrow \infty} \frac{1}{\mu} \log \tilde{\sigma}_{\delta}^{\star}(\lambda, \mu)=\lim _{\mu \rightarrow \infty} \frac{1}{\mu} \log \hat{\sigma}_{\rho, \delta}^{\star}(\lambda, \mu)=S_{c}(\lambda) .
$$

At first sight, the results of our theorems can be found quite unsatisfactory, because they give us only the estimation in logarithmic scale and in the limit of the strong disorder. However, they can be useful to find out the dependence of the effective conductivity on other parameters. Indeed, let the local conductivity $\sigma(x, \alpha)$ be defined by $\exp (\mu f(S(x), \alpha)$ ), where $f$ is a strictly increasing and differentiable in the first argument, and with the first derivative with respect to this argument in the point $S_{c}(\lambda)$ bounded away from zero and infinity. Then an easy modification of the arguments given in the proof of Theorem 2.1 gives

$$
\lim _{\mu \rightarrow \infty} \frac{1}{\mu} \log \sigma^{\star}(\lambda, \mu, \alpha)=f\left(S_{c}(\lambda), \alpha\right) .
$$

This is essentially the way how the idea of CPA was used in the original article [1].

Note also that there are two reasons for having results only in the logarithmic scale. The first one is the "nongaussian" shape of the graph of the conductivity around the saddle points. This problem can be probably resolved by a more careful computation. However, there is still a second problem. We do not have enough control of the infinite cluster of continuum percolation near the critical point.

The proofs of Theorems 2.1 and 2.2 can be found in Section 4 and they use homogenisation techniques. In Section 3 we show some facts about continuum percolation in $\mathbb{R}^{2}$. 


\section{Percolation results}

In this section we prove some facts that are known to be valid for discrete percolation. To our knowledge similar results do not exist in the case of continuum percolation. The proofs we present are rather standard modifications of the discrete versions. The reader familiar with the technical details can skip the rest of this section and read only Propositions 3.1 and 3.7 that will be used later.

Let $N$ be a set of all finite counting measures assigning the weight at most one to singletons equipped with the usual $\sigma$-field $\mathcal{N}$ generated by sets of the form $\{n \in N: n(A)=k\}$, where $A \subset \mathbb{R}^{2}$ is a Borel set and $k \in \mathbb{N}$. Every $n \in N$ can be identified with a set of points in $\mathbb{R}^{2}$. This allows us to write $x \in n$, if $n$ has an atom at $x \in \mathbb{R}$.

Let $(\Omega, \mathcal{F}, \mathbb{P})$ be some probability space. The Poisson point process with density $\lambda$ is an $N$-valued random variable which satisfies the following two conditions. $X(A)$ is a Poisson random variable with mean $\lambda|A|$, where $|A|$ denotes the Lebesgue measure of $A$. If $A_{1}, A_{2} \subset \mathbb{R}^{2}, A_{1} \cap A_{2}=\emptyset$, then $X\left(A_{1}\right)$ and $X\left(A_{2}\right)$ are independent. We write $\mathbb{P}_{\lambda}$ for the law of $X$ and $\mathbb{E}_{\lambda}$ for the corresponding expectation.

Let us now define set $\mathbb{X}(\omega), \omega \in \Omega$, as the set $\left\{x \in \mathbb{R}^{2}: S(x) \leqslant 1\right\}$. The set $\mathbb{X}$ is the union of unit disks with centres in $X(\omega)$. We will call it the occupied region. The complement of $\mathbb{X}(\omega)$ is called the vacant region. For any $A \subset \mathbb{R}^{2}$ we use $W(A)$ to denote the union of all components of $\mathbb{X}$ (occupied components) intersecting $A$. Similarly, we write $V(A)$ for the union of vacant components intersecting $A$. It is well known that in dimension two there exists a constant $\lambda_{c}$ such that for every bounded set $A$ the following holds

$$
\begin{aligned}
\lambda_{c} & =\sup \left\{\lambda: \mathbb{P}_{\lambda}[\operatorname{diam} V(A)=\infty]>0\right\}=\inf \left\{\lambda: \mathbb{E}_{\lambda}[\operatorname{diam} V(A)]<\infty\right\} \\
& =\inf \left\{\lambda: \mathbb{P}_{\lambda}[\operatorname{diam} W(A)=\infty]>0\right\}=\sup \left\{\lambda: \mathbb{E}_{\lambda}[\operatorname{diam} W(A)]<\infty\right\},
\end{aligned}
$$

i.e. occupied region percolates above $\lambda_{c}$ and vacant region percolates below $\lambda_{c}$.

Let $E$ be an event. We say that $E$ is increasing event if from $\omega \in E$ follows $\omega^{\prime} \in E$ for all $\omega^{\prime}$ satisfying $X\left(\omega^{\prime}\right) \supset$ $X(\omega)$. The event $E$ is decreasing if $E^{c}$ is increasing.

We now introduce some obvious geometrical notation. Let $A_{1}, A_{2}, B$ be subsets of $\mathbb{R}^{2}$. We write $A_{1} \underset{\text { in } B}{\stackrel{\text { occ }}{\longrightarrow}} A_{2}$ if $A_{1}$ is connected to $A_{2}$ in $B \cap \mathbb{X}$, i.e. there exists a continuous function $\phi:[0,1] \mapsto \mathbb{R}^{2}$ such that $\phi(0) \in A_{1}$, $\phi(1) \in A_{2}$, and $\phi(t) \in \mathbb{X} \cap B$ for every $t \in[0,1]$. If the set $B$ is omitted, then it is understood $B=\mathbb{R}^{2}$. We use $A_{1} \underset{\text { out } B}{\stackrel{\text { occ }}{\longrightarrow}} A_{2}$ for $A_{1} \underset{\text { in } B^{c}}{\stackrel{\text { occ }}{\longrightarrow}} A_{2}$. Similarly, we write $A_{1} \underset{\text { in } B}{\stackrel{\text { vac }}{\longrightarrow}} A_{2}$ if there exists a curve connecting $A_{1}$ and $A_{2}$ laying completely in $B \cap \mathbb{X}^{c}$.

Let $B_{L}(x)$ be the box $\left[x^{1}-L, x^{1}+L\right] \times\left[x^{2}-L, x^{2}+L\right]$. We say that the polygonal line $x_{i}, i=0, \ldots, n$, forms a left-right (LR) occupied crossing of $B_{L}(0)$ if all points $x_{i}$ are in $X$, the disks around the successive points intersect (i.e. $\left.d\left(x_{i-1}, x_{i}\right) \leqslant 2, i=1, \ldots, n\right)$, the points $x_{i}, i=1, \ldots, n-1$, are in $B_{L}(0)$, and the first and the last disk intersect the left, resp. right, edge of $B_{L}(0)$ (i.e. $x_{0}^{1} \in[-L-1,-L+1], x_{n}^{1} \in[L-1, L+1]$ ). Two LR occupied crossings are called disjoint if the corresponding polygonal lines do not intersect.

A smooth curve $\phi:[0,1] \mapsto \mathbb{R}^{2}$ is called LR vacant crossing of $B_{L}(0)$ if $\phi(0) \in\{-L\} \times[-L, L], \phi(1) \in$ $\{L\} \times[-L, L]$, and $\phi([0,1]) \in B_{L}(0) \cap \mathbb{X}^{c}$. Two LR vacant crossings $\phi$ and $\phi^{\prime}$ are called disjoint if

$$
\inf \left\{d\left(\phi(t), \phi^{\prime}\left(t^{\prime}\right)\right): t, t^{\prime} \in[0,1]\right\} \geqslant 2 .
$$

The constant 2 has not any particular importance, any other positive constant can be chosen. Similarly, one defines the top-bottom (TB) crossings of $B_{L}(0)$. We will need the following proposition to prove Theorem 2.1.

\section{Proposition 3.1.}

(a) Let $\lambda>\lambda_{c}$, then there exist positive constants $\beta, \gamma, L_{0}$ depending only on $\lambda$ such that

$$
\mathbb{P}_{\lambda}\left[\# \text { of disjoint occ. LR crossings of } B_{L}(0) \leqslant \beta L\right] \leqslant \mathrm{e}^{-\gamma L}
$$

for $L \geqslant L_{0}$. 
(b) Let $\lambda<\lambda_{c}$, then there exist positive constants $\beta^{\prime}, \gamma^{\prime}, L_{0}^{\prime}$ depending only on $\lambda$ such that

$$
\mathbb{P}_{\lambda}\left[\# \text { of disjoint vac. LR crossings of } B_{L}(0) \leqslant \beta^{\prime} L\right] \leqslant \mathrm{e}^{-\gamma^{\prime} L}
$$

for $L \geqslant L_{0}^{\prime}$.

We will prove part (a) of this proposition using the methods that are strongly inspired by discrete percolation (see [5, Lemma 11.22]). We start with the following lemma.

Lemma 3.2. Let $\lambda>\lambda_{c}$, then there exists $\kappa>0$, such that for $L$ large enough

$\mathbb{P}_{\lambda}\left[\exists\right.$ occ. LR crossing of $\left.B_{L}(0)\right] \geqslant 1-\mathrm{e}^{-\kappa L}$.

Proof. Using duality in $\mathbb{R}^{2}$ it is easy to see

$\mathbb{P}_{\lambda}\left[\nexists\right.$ occ. LR crossing of $\left.B_{L}(0)\right]=\mathbb{P}_{\lambda}\left[\exists\right.$ vac. TB crossing of $\left.B_{L}(0)\right]$.

If we place on the upper edge of $B_{L}(0) 2 L+1$ boxes of size 2, then it is easy to see that the last expression can be bounded by

$$
\begin{aligned}
& \leqslant \sum_{i=-L}^{L} \mathbb{P}_{\lambda}\left[B_{1}((i, L)) \stackrel{\mathrm{vac}}{\longleftrightarrow} \text { lower edge of } B_{L}(0)\right] \\
& \leqslant(2 L+1) \mathbb{P}_{\lambda}\left[B_{1}(0) \stackrel{\mathrm{vac}}{\longleftrightarrow} \partial B_{2 L}(0)\right] .
\end{aligned}
$$

We used the obvious notation $\partial B_{L}(0)$ for boundary of $B_{L}(0)$ and the translation invariance of the measure $\mathbb{P}_{\lambda}$.

Since $\lambda>\lambda_{c}$, it follows from (12) that $\mathbb{E}_{\lambda}\left[\operatorname{diam}\left(V\left(B_{1}(0)\right)\right)\right]<\infty$. Denoting by $\operatorname{diam}^{\prime}(A)$ the diameter of the set $A$ in $\infty$-norm and using the obvious fact $\operatorname{diam}^{\prime}(A) \leqslant \operatorname{diam}(A)$, we can write

$$
\begin{aligned}
\infty & >\mathbb{E}_{\lambda}\left[\operatorname{diam}\left(V\left(B_{1}(0)\right)\right)\right] \geqslant \mathbb{E}_{\lambda}\left[\operatorname{diam}^{\prime}\left(V\left(B_{1}(0)\right)\right)\right] \geqslant \mathbb{E}_{\lambda}\left[\sup \left\{\|x\|_{\infty}: x \in V\left(B_{1}(0)\right)\right\}\right] \\
& \geqslant \sum_{i=0}^{\infty} \mathbb{P}_{\lambda}\left[\sup \left\{\|x\|_{\infty}: x \in V\left(B_{1}(0)\right)\right\} \geqslant i\right]=\sum_{i=0}^{\infty} \mathbb{P}_{\lambda}\left[B_{1}(0) \stackrel{\mathrm{vac}}{\longleftrightarrow} \partial B_{i}(0)\right] .
\end{aligned}
$$

From the last expression one can see that there exist $k$ such that

$$
4(k+2) \mathbb{P}\left(0 \stackrel{\mathrm{vac}}{\longleftrightarrow} \partial B_{k}(0)\right) \leqslant \eta<1 .
$$

Indeed, suppose on the contrary that $P\left(0 \stackrel{\mathrm{vac}}{\longleftrightarrow} \partial B_{k}(0)\right)>\eta / 4(k+2)$ for every $k$. Then the last sum in (19) is clearly infinite and we get the contradiction with the first inequality in (19).

Let $N \geqslant k+2$. By dividing the vacant connection from 0 to $\partial B_{N}(0)$ into two parts, first one from 0 to $\partial B_{k}(0)$ and second one from $\partial B_{k+2}(0)$ to $\partial B_{N}(0)$ we get

$$
\mathbb{P}_{\lambda}\left[B_{1}(0) \stackrel{\mathrm{vac}}{\longleftrightarrow} \partial B_{N}(0)\right] \leqslant \mathbb{P}_{\lambda}\left[\left(B_{1}(0) \stackrel{\mathrm{vac}}{\longleftrightarrow} \partial B_{k}(0)\right) \cap\left(\partial B_{k+2}(0) \underset{\text { out } \stackrel{\mathrm{vac}}{B_{k+2}(0)}}{\longleftrightarrow} \partial B_{N}(0)\right)\right] .
$$

Further, let $\mathcal{Z}$ be the set of points laying on the segments composing the boundary of $B_{k+2}$ that have the distance from the vertices of these segments divisible by 2 . Around every point of $\mathcal{Z}$ we put a box whose edges have length 2 . We get

$$
\mathbb{P}_{\lambda}\left[B_{1}(0) \stackrel{\mathrm{vac}}{\longleftrightarrow} \partial B_{N}(0)\right] \leqslant \mathbb{P}_{\lambda}\left[B_{1}(0) \stackrel{\mathrm{vac}}{\longleftrightarrow} \partial B_{k}(0) \cap\left(\bigcup_{z \in \mathcal{Z}} B_{1}(z) \underset{\text { out } \stackrel{\mathrm{vac}}{\longleftrightarrow}}{B_{k+2}(0)} \partial B_{N}(0)\right)\right] .
$$

The events in the last equation are decreasing and are chosen to be disjoint (i.e. the disks, that can have influence on the first event cannot change the second and vice versa). We can thus use BK inequality proved for continuum percolation in [6]. Hence, 


$$
\begin{aligned}
\mathbb{P}_{\lambda}\left[B_{1}(0) \stackrel{\mathrm{vac}}{\longleftrightarrow} \partial B_{N}(0)\right] & \leqslant \mathbb{P}_{\lambda}\left[B_{1}(0) \stackrel{\mathrm{vac}}{\longleftrightarrow} \partial B_{k}(0)\right] \sum_{z} \mathbb{P}_{\lambda}\left[B_{1}(z) \underset{\text { out } B_{k+2}(0)}{\stackrel{\mathrm{vac}}{\longleftrightarrow}} \partial B_{N}(0)\right] \\
& \leqslant 4(k+2) \mathbb{P}_{\lambda}\left[B_{1}(0) \stackrel{\mathrm{vac}}{\longleftrightarrow} \partial B_{k}(0)\right] \mathbb{P}_{\lambda}\left[B_{1}(0) \stackrel{\mathrm{vac}}{\longleftrightarrow} \partial B_{N-k-2}(0)\right] \\
& \leqslant \eta \mathbb{P}_{\lambda}\left[B_{1}(0) \stackrel{\mathrm{vac}}{\longleftrightarrow} \partial B_{N-k-2}(0)\right] .
\end{aligned}
$$

We used again the translation invariance of $\mathbb{P}_{\lambda}$ and (20). Iterating Eq. (23) until $N-j(k+2) \geqslant k+2$ we get

$$
\mathbb{P}_{\lambda}\left[B_{1}(0) \stackrel{\text { vac }}{\longleftrightarrow} \partial B_{N}(0)\right] \leqslant \eta^{\lfloor N /(k+2)\rfloor} .
$$

Substituting this into (18) we obtain

$$
\mathbb{P}_{\lambda}\left[\nexists \text { occ. LR crossing of } B_{L}(0)\right] \leqslant(2 L+1) \eta^{\lfloor 2 L /(k+2)\rfloor}
$$

and the proof is finished taking $L$ sufficiently large and $\kappa$ slightly smaller than $-2 \log \eta /(k+2)>0$.

To state the next lemma we need the following definition. Let $E$ be an increasing event. We define the $r$-kernel $I_{r}(E)$ of this event as $I_{r}(E)=\left\{\omega \in E\right.$ : every $\omega^{\prime}$ such that $X(\omega) \supset X\left(\omega^{\prime}\right)$ and $\left|X(\omega) \backslash X\left(\omega^{\prime}\right)\right| \leqslant r$ is also in $\left.E\right\}$. The event $I_{r}(E)$ is the set of configurations from which we can delete arbitrary $r$ disks and $E$ still occurs. The utility of this definition follows from the fact that the $r$-kernel of the event "there is a LR occupied crossing" is the event "there are $r+1$ LR occupied crossings". We have the following lemma (compare it with [5, Theorem 2.45]).

Lemma 3.3. Let $\lambda_{2}>\lambda_{1}$ and let $E$ be an increasing event. Then

$$
1-\mathbb{P}_{\lambda_{2}}\left[I_{r}(E)\right] \leqslant\left(\frac{\lambda_{2}}{\lambda_{2}-\lambda_{1}}\right)^{r}\left(1-\mathbb{P}_{\lambda_{1}}[E]\right)
$$

Proof. Let $X^{\prime}$ be the $\lambda_{1} / \lambda_{2}$-thinning of $X$, i.e. the point process that we obtain from $X$ by deleting each point independently with probability $1-\lambda_{1} / \lambda_{2}$. If $X$ is the Poisson point process with density $\lambda_{2}$, then $X^{\prime}$ is again a Poisson point process, but this time with density $\lambda_{1}$. If $\omega \notin I_{r}(E)$, then there exists a set $B \subset X(\omega)$, such that $|B| \leqslant r$ and $\tilde{\omega}$ obtained from $\omega$ by deleting the points in $B$ is not in $E$. If there are more such sets $B$, we choose one according to some predefined order. Conditionally on $B$, there is probability $\left(1-\lambda_{1} / \lambda_{2}\right)^{|B|}$ that we delete all points in $B$, i.e. we have

$$
\begin{aligned}
& \mathbb{P}\left[X^{\prime} \notin E \mid X \notin I_{r}(E)\right] \geqslant\left(1-\frac{\lambda_{1}}{\lambda_{2}}\right)^{r}, \\
& \mathbb{P}\left[X^{\prime} \notin E\right] \geqslant\left(\frac{\lambda_{2}-\lambda_{1}}{\lambda_{2}}\right)^{r} \mathbb{P}\left[X \notin I_{r}(E)\right]
\end{aligned}
$$

and the claim follows easily.

Proof of Proposition 3.1(a). Let $A_{L}$ be the event that there exists an occupied LR crossing of $B_{L}(0)$. If $\lambda>\lambda_{c}$, then there exists $\lambda^{\prime}$, such that $\lambda>\lambda^{\prime}>\lambda_{c}$ and $\kappa>0$, such that

$$
P_{\lambda^{\prime}}\left[A_{L}\right] \geqslant 1-\mathrm{e}^{-\kappa L} \quad \text { for } L \geqslant L_{0} .
$$

Since $I_{r}\left(A_{L}\right)=\{\exists$ at least $r+1$ disjoint LR occupied crossings $\}$ we choose $r=\beta L$. Using Lemma 3.3 we have

$$
1-\mathbb{P}_{\lambda}[\exists \text { at least } \beta L \text { occ. LR crossings }] \leqslant\left(\frac{\lambda}{\lambda-\lambda^{\prime}}\right)^{\beta L} \mathrm{e}^{-\kappa L} .
$$

We now take $\beta$ small enough to have $\gamma\left(\lambda, \lambda^{\prime}, \beta\right)=\kappa\left(\lambda^{\prime}\right)-\beta \log \frac{\lambda}{\lambda-\lambda}>0$. Using this choice we easily complete the proof. 
Proof of Proposition 3.1(b). The proof of this part is slightly more complicated since the vacant crossings do not have the discrete underlying structure. We will use a coarse graining to reduce this case to the discrete site percolation. We start with the following lemma.

Lemma 3.4. Let $H(M, L)$ be the event that there is vacant crossing of the rectangle with sides $M$ and $2 L$ connecting the sides with length $M$. If $\lambda<\lambda_{c}$, then there exist positive constants $C, \rho$ such that

$$
\mathbb{P}_{\lambda}[H(M, L)] \geqslant 1-C L \mathrm{e}^{-\rho M} .
$$

\section{Proof.}

$$
\begin{aligned}
\mathbb{P}_{\lambda}[H(M, L)] & =1-\mathbb{P}_{\lambda}[\exists \text { occ. crossing in perpendicular direction }] \\
& \leqslant 1-2 L \mathbb{P}_{\lambda}\left[0 \stackrel{\text { occ }}{\longleftrightarrow} \partial B_{M}(0)\right] \leqslant 1-2 L C \mathrm{e}^{-\rho M} .
\end{aligned}
$$

In the last inequality we use the fact that if $\lambda<\lambda_{c}$, then (see page 38 of [10])

$$
\mathbb{P}_{\lambda}\left(0 \stackrel{\text { occ }}{\longleftrightarrow} \partial B_{M}(0)\right) \leqslant C \mathrm{e}^{-\rho M} .
$$

This finishes the proof.

Using this lemma we will prove a two-dimensional version of coarse graining following closely the proof from [5, p. 191]. We call the box $B_{k}(x)$ good if the next two conditions hold:

(i) there are both TB and LR vacant crossings of $B_{k}(x)$;

(ii) all other vacant clusters have diameter (in $\infty$-norm) smaller than $k$.

We want to prove the following lemma.

Lemma 3.5. If $\lambda<\lambda_{c}$, then for every $\varepsilon>0$ there exist $k$, such that

$$
\mathbb{P}\left[B_{k}(x) \text { is good }\right] \geqslant 1-\varepsilon .
$$

Proof. Without lost of generality we put $x=0$. Let $\rho$ be the constant from Lemma 3.4, $v>1 / \rho$, and $k$ large enough such that $v \log k \leqslant k$. We take four rectangles with sides $2 k$ and $\nu \log k$ composing an "annulus" around the origin with the "outer radius" $k$ and "inner radius" $k-v \log k$. More precisely, let $R_{1}$ be the rectangle $[-k, k] \times$ [ $-k,-k+v \log k]$ and let $R_{2}, R_{3}$ and $R_{4}$ be its images under rotations by $\pi / 2, \pi$, and $3 \pi / 2$ around the origin.

Let $B$ denote the event that there is a vacant crossing connecting the sides of length $v \log k$ inside of all these rectangles. The probability of this event can be bounded from below using the FKG inequality,

$$
\mathbb{P}_{\lambda}[B] \geqslant\left(\mathbb{P}_{\lambda}[H(v \log k, k)]\right)^{4} .
$$

Applying the previous lemma we have

$$
\mathbb{P}_{\lambda}[B] \geqslant\left(1-A k^{1-\rho \nu}\right)^{4} .
$$

The last expression converges to 1 as $k$ goes to infinity. Hence, we verified that condition (i) from the definition of the good block can be satisfied with arbitrarily large probability.

It remains to exclude the possibility that there is another cluster with diameter larger than $k$. This cluster has to cross the rectangle $[-k, k] \times[i, i+k]$ vertically or $[i, i+k] \times[-k, k]$ horizontally $(-k \leqslant i \leqslant 0)$. However, the probability that there is horizontal or vertical vacant crossing of this rectangle turns exponentially to 1 . Hence, this cluster is with overwhelming probability connected to the vacant crossing of one of the four rectangles $R_{1}, \ldots, R_{4}$. This finishes the proof of Lemma 3.5. 
We now construct a block process $Z_{x}, x \in \mathbb{Z}^{2}$. Let $\varepsilon>0$ and choose $k$ large enough such that $\mathbb{P}_{\lambda}\left[B_{k}(0)\right.$ is good] $\geqslant 1-\varepsilon$. Let $Z_{x}=1$ if $B_{k}(x k)$ is good, and $Z_{x}=0$ otherwise. Obviously, $Z_{x}$ is a dependent site percolation on $\mathbb{Z}^{2}$ with probability that $Z_{x}=1$ larger than $1-\varepsilon$. The definition of the good blocks implies the following property. For every nearest neighbours path $x_{1}, \ldots, x_{n}$ in $\mathbb{Z}^{2}$ such that $Z_{x_{i}}=1, i=1, \ldots, n$, there exist a vacant path of original continuum percolation passing through the blocks $B_{k}\left(x_{i} k\right)$. Hence, if we show that there is at least $\beta L$ disjoint crossings of the square $B_{L / k}(0)$ for the process $Z_{x}$, the proof will be finished.

To prove this we use the standard method, namely stochastic domination. Let $U_{x}$ and $V_{x}$ be two families of random variables indexed by $x \in \mathbb{Z}^{2}$ and taking values in the set $\{0,1\}$. We say that $U$ stochastically dominates $V$ if for all bounded, increasing, measurable functions $f:\{0,1\}^{\mathbb{Z}^{2}} \rightarrow \mathbb{R}$ we have

$$
\mathbb{E}(f(U)) \geqslant \mathbb{E}(f(V)) .
$$

We say that the family $U_{x}$ is $k$-dependent if the random variables $U_{x}$ and $U_{y}$ are independent for all $x, y$ such that $\|x-y\|_{\infty}>k$. The block process $Z_{x}$ is clearly 2-dependent. Let $Y_{x}^{p}$ denote the independent Bernoulli site percolation process on $\mathbb{Z}^{2}$ with the density $p$ and let $\mathbb{P}_{p}^{\star}$ denote its measure. We use the following lemma from [9].

Lemma 3.6. Let $V_{x}$ be a $k$-dependent family of random variables that satisfies $\mathbb{P}\left[V_{x}\right] \geqslant \delta$ for all $x \in \mathbb{Z}^{2}$. Then there exists a non-decreasing function $\pi(\delta):[0,1] \rightarrow[0,1]$ satisfying $\pi(\delta) \rightarrow 1$ as $\delta \rightarrow 1$, such that $V$ stochastically dominates $Y^{\pi(\delta)}$.

We apply this lemma with $V=Z$. Let $C$ be the event "there is at least $\beta L$ disjoint LR crossings of $B_{L / k}(0)$ ". The event $C$ is clearly increasing. Thus we have

$$
\mathbb{P}_{\lambda}(C) \geqslant \mathbb{P}_{\pi(1-\varepsilon)}^{\star}(C) \text {. }
$$

We take $\varepsilon$ such that $\pi(1-\varepsilon)$ is larger than the percolation threshold $p_{c}$ of independent site percolation. It is known that for independent site percolation above the threshold there exist constants $\tilde{\beta}$ and $\tilde{\gamma}$ such that

$$
\mathbb{P}_{p}^{\star}\left[\text { there is at least } \tilde{\beta} L \text { crossings of } B_{L}(0)\right] \geqslant 1-\mathrm{e}^{-\tilde{\gamma} L} \text {. }
$$

Using this fact we easily complete the proof.

For the proof of Theorem 2.2 we will need the following proposition. We recall that $\mathcal{V}$ denotes the set of borders of Voronoi cells around the points of the point process $X$. Let $\mathcal{W} \subset \mathcal{V}$. The LR crossing of $B_{L}(0)$ in $\mathcal{W}$ is the curve $\phi:[0,1] \rightarrow \mathbb{R}^{2}$ connecting the left and right side of $B_{L}(0)$ such that $\phi([0,1])$ is a subset of $\mathcal{W} \cap B_{L}(0)$. Two LR crossings are disjoint if they do not intersect.

Proposition 3.7. Let $\mathcal{W}$ be the set of bonds $b$ in $\mathcal{V}$ such that $d(b, X) \geqslant 1$ and let $0<\lambda<\lambda_{c}$. Then there exist positive constants $\beta^{\prime \prime}, \gamma^{\prime \prime}$ and $L_{0}^{\prime \prime}$ depending only on $\lambda$ such that

$$
\mathbb{P}_{\lambda}\left[\# \text { of disjoint } L R \text { crossings of } B_{L}(0) \text { in } \mathcal{W} \leqslant \beta^{\prime \prime} L\right] \leqslant \mathrm{e}^{-\gamma^{\prime \prime} L}
$$

for $L \geqslant L_{0}^{\prime \prime}$.

Proof. The proof of this proposition can be probably done by more elementary methods, but we prefer to use the previous result to prove it. We will use the fact that for every vacant crossing of $B_{L}(0)$ it is possible to find a path in $\mathcal{W}$ that is "not far" from this crossing.

To formalise the previous claim we first define the equivalence relation between LR vacant crossings of the strip $\mathcal{S}_{L}=[-L, L] \times \mathbb{R}$ (the LR vacant crossings of $\mathcal{S}_{L}$ are defined in the obvious way). We say that two crossings $\phi_{1}$ and $\phi_{2}$ are equivalent if there exists a continuous function $\Phi(t, s)$, such that $\Phi(t, 0)=\phi_{1}(t), \Phi(t, 1)=\phi_{2}(t)$, for every fixed $s \in[0,1] \Phi(t, s)$ is a LR crossing of $\mathcal{S}_{L}$, and $\Phi([0,1] \times[0,1]) \cap X=\emptyset$. Less formally, two crossings are not equivalent if there is a disk between them. 


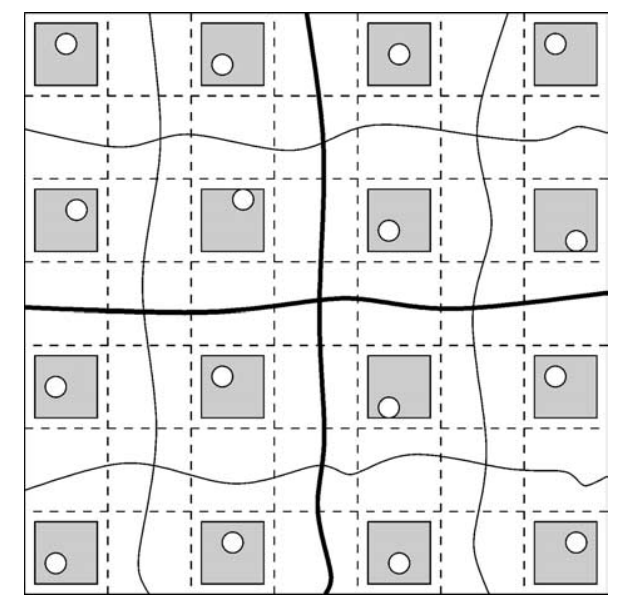

Fig. 1. Good block.

Observing now that every component $W$ of the occupied region $\mathbb{X}$ is separated from $\mathbb{X} \backslash W$ by a loop in $\mathcal{W}$, it is easy to see that every vacant LR crossing of $B_{L}(0)$ is equivalent to a path in $\mathcal{W}$ that forms a crossing of $\mathcal{S}_{L}$ and, moreover, this path is almost uniquely determined (up to its starting and ending parts). There are two problems with this path. First, it can leave the box $B_{L}(0)$, secondly, two disjoint occupied crossings can be transformed to not disjoint paths in $\mathcal{W}$. Hence, we should construct a sufficient number of vacant crossings such that these two cases do not happen.

This can be achieved by a redefinition of the good blocks. We want to assure that the vacant crossing of the good block does not leave it after the transformation to a path in $\mathcal{W}$ and that the crossings of two neighbouring good blocks cannot be equivalent. The easiest way how to achieve it, is to force the good blocks to contain some disks that will force the paths in $\mathcal{W}$ to stay in the box. One way to do it is to consider the following definition of the good block.

We say that the block $B_{7 k}(0)$ is good if every rectangle $[(2 j-1) k,(2 j+1) k] \times[-7 k, 7 k], j \in\{-2,0,2\}$ contains a vertical vacant crossing and every rectangle $[-7 k, 7 k] \times[(2 j-1) k,(2 j+1) k]$ contains a horizontal vacant crossing. More over, every square

$$
G_{j l}=[(2 j-1) k+1,(2 j+1) k-1] \times[(2 l-1) k+1,(2 l+1) k-1],
$$

where $j, l \in\{-3,-1,1,3\}$, contains at least one disk. This construction is illustrated on Fig. 1.

The reader can verify that the disks in the squares $G_{j l}$ do not permit the paths in $\mathcal{W}$ equivalent to the crossings of $[-k, k] \times[-7 k, 7 k]$ and $[-7 k, 7 k] \times[-k, k]$ to leave the box $B_{7 k}(0)$. We define the box $B_{7 k}(x)$ being good in the obvious way.

We should now show that the probability of the block being good can be made arbitrarily close to one. First, we observe that the crossings of the rectangles are independent of the configuration of $X$ in the squares $G_{j l}$. The probability of having the long vacant crossings in all six rectangles can be bounded from below using the FKG inequality and Lemma 3.4 by $(1-7 C k \exp (-2 k))^{6}$. The probability that there is at least one disk in any of $G_{j l}$ is $1-\exp \left(-\lambda(2 k-2)^{2}\right)$. Hence

$$
\mathbb{P}\left(B_{7 k}(x) \text { is good }\right) \geqslant(1-7 C k \exp (-2 k))^{6}\left[1-\exp \left(-\lambda(2 k-2)^{2}\right)\right]^{16} .
$$

Taking $k$ large enough the right hand side of the previous expression can be made arbitrarily close to one.

We proceed in the obvious way. We define the process $Z_{x}, x \in \mathbb{Z}^{2}$. We set $Z_{x}=1$ if the block $B_{k}(k x)$ is good. Otherwise we set $Z_{x}=0$. As before, having path in $Z$ assures us to have a crossing in $\mathcal{W}$ not leaving the boxes corresponding to the points of this path. Then we can continue exactly in the same manner as in the proof of Proposition 3.1. 


\section{Proof of Theorems 2.1 and 2.2}

Proof of Theorem 2.1. To prove Theorem 2.1 we apply the usual strategy. We express the effective conductivity $\sigma^{\star}(\lambda, \mu)$ in the form of a variational formula and we construct a test function that plugged into it will give us the required bound.

Upper bound: We use the following formula

$$
\sigma^{\star}=\lim _{N \rightarrow \infty} \frac{1}{N^{2}} \inf _{u \in \mathcal{P}} \int_{\Lambda_{N}} \sigma(x)|\nabla u(x)|^{2} \mathrm{~d} x,
$$

where $\Lambda_{N}=[0, N]^{2}$ and

$$
\mathcal{P}=\left\{u \in H^{1}\left(\Lambda_{N}\right): u \text { satisfies the boundary conditions in (4) }\right\} .
$$

The infimum in (42) is attained by the solution of the system (4). That is why we are looking for a function that is not far from the solution and, moreover, the integral on the right-hand side of (42) is easy to compute.

Using the one-dimensional analogy of our problem, it is not difficult to check that the potential $u$ has large gradient in the places where there is a barrier to go through, i.e. where the conductivity is small. In the twodimensional case such barriers should span all the width of the box. As we have already noted, our medium can be regarded as an ensemble of insulating grains around the points of the point process $X$. Hence, the easiest way how to construct a barrier is to have a chain of closely packed grains crossing the box from the top to the bottom. We need to specify what we mean by "closely packed". According to the definition of $S_{c}(\lambda)$ we could not expect to find a crossing of the large box with the grains that have centres at a distance smaller than $2 S_{c}(\lambda)$. Thus, we will choose the radius of grains slightly larger than $S_{c}(\lambda)$.

Let take $\varepsilon>0$ and consider grains with the radius $S_{c}(\lambda)+\varepsilon$. We rescale temporarily the box $\Lambda_{N}$ such that these grains become disks with radius 1 . After the scaling we get a point process with density

$$
\lambda^{\prime}=\lambda\left(S_{c}(\lambda)+\varepsilon\right)^{2} .
$$

From the definition (12) of $\lambda_{c}$ it is easy to see that

$$
S_{c}\left(\lambda_{c}\right)=1 .
$$

Another application of scaling properties of the Poisson point process gives us

$$
\lambda S_{c}(\lambda)^{2}=\lambda_{c} .
$$

If we put together the last three claims, we get $\lambda^{\prime}>\lambda_{c}$. According to Proposition 3.1(a), we know that there are with overwhelming probability at least

$$
\beta\left(\lambda^{\prime}\right) N\left(2\left(S_{c}(\lambda)+\varepsilon\right)\right)^{-1} \equiv \beta_{\varepsilon} N
$$

top-bottom occupied crossings of rescaled box $\Lambda_{N}$ with disks of radius one. If we now return to the original scale, we obtain $\beta_{\varepsilon} N$ chains of disks with radius $S_{c}(\lambda)+\varepsilon$ crossing $\Lambda_{N}$. Note that it will become clear in the next part of the proof why we need $\mathrm{O}(N)$ crossings. One crossing would not be sufficient for our purposes.

We now define the test function that we will use. We use $S_{i}$ to denote the crossings which we discussed in the previous paragraph. Let $i=1, \ldots, R$, with $R$ being the random number of crossings. We denote the crossings in the way that $S_{1}$ is the left most one, $S_{2}$ the second left one, etc. We recall that the occupied crossing was defined as a sequence of points from $X$ with certain properties. We use $x_{j}^{(i)}, j=1, \ldots, n_{i}$, to denote the points composing $S_{i}$ in the way that $x_{1}^{(i)}$ is the point that is close to the lower edge and $x_{n_{i}}^{(i)}$ is close to the upper edge of $\Lambda_{N}$. We use $\bar{S}_{i}$ to denote the polygonal line connecting them. When $x_{1}^{(i)}$ is in the interior of $\Lambda_{N}$, we extend $\bar{S}_{i}$ by the vertical segment connecting $x_{1}^{(i)}$ with the lower edge of $\Lambda_{N}$. Similarly, if $x_{n_{i}}^{(i)}$ is in the interior of $\Lambda_{N}$, we connect it to the upper edge. Now, every line $\bar{S}_{i}$ divides the box into two disjoint parts. 
We continue by smoothing off the lines $\bar{S}_{i}$. By smoothing we mean replacing the curves $\bar{S}_{i}$ by other set of curves that will be everywhere once differentiable and will have bounded curvature. The smoothing is necessary, it allows to construct a test-function that will have well defined gradient everywhere around these curves. The way how the smoothing is defined has no particular importance. For the sake of definiteness we chose the following one.

We will change the curves $\bar{S}_{i}$ only in the neighbourhoods $U\left(x_{j}^{(i)}\right)$ of $x_{j}^{(i)}$ with the radius $S_{c}(\lambda) / 10$. Choose one such point $x$. If there is no $y \in S_{i}$ such that $U(x) \cap U(y) \neq \emptyset$, we simply replace the two segments of $\bar{S}_{i}$ in $U(x)$ by a piece of circle. We do it in the way that the resulting curve is everywhere once differentiable. Since we can suppose that the minimal angle by any point $x_{j}^{(i)} \in S_{i}$ is $\pi / 3$ (otherwise we can connect directly $x_{j-1}^{(i)}$ with $x_{j+1}^{(i)}$ ), we can bound the radius of the circle from below by some positive constant.

If, on the other hand, there is vertex $y \in S_{i}$ satisfying $U(x) \cap U(y) \neq \emptyset$, we argue in the following way. First, note that we can "optimise" the sets $S_{i}$ in the way that for every point $x$ there is at most one such $y$. Hence, we can consider only the pairs of "close" vertices. We should replace the polygonal line in the union of neighbourhoods $U(x), U(y)$ by a smooth curve. We let the reader check that it is possible to make such replacement by two pieces of circle with the radii bounded from below.

Finally, we deform $\bar{S}_{i}$ slightly at its ends in the way that the smooth version is perpendicular to the boundary of $\Lambda_{N}$. We denote the smooth version of $\bar{S}_{i}$ by $\tilde{S}_{i}$. We use $c_{r}$ to denote the lower bound on the radius of curvature of $\tilde{S}_{i}$.

Let us choose another constant $0<d<c_{r}$. Denote by $S_{i}$ the "tube" of radius $d$ around $\tilde{S}_{i}$, i.e. the set $\{x \in$ $\left.\Lambda_{N}: d\left(x, \tilde{S}_{i}\right) \leqslant d\right\}$. We use $S_{i}^{L}, S_{i}^{R}$ to denote left and right boundary of $S_{i}$. Let $S_{0}^{R}$, resp. $S_{R+1}^{L}$, be the left, resp. right, edge of $\Lambda_{N}$.

We construct the test function $u^{\star}(x)$ as follows. Let $u^{\star}(x)$ be constant between $S_{i}^{R}$ and $S_{i+1}^{L}, i=0, \ldots, R$, and let $u^{\star}(x)$ grow linearly on the segments perpendicular to $\tilde{S}_{i}$ in the tubes $S_{i}$. The condition $d<c_{r}$ ensures that for any point in $S_{i}$ there is one and only one such segment. Let $u^{\star}(x)$ be continuous in $\Lambda_{N}$ and let the difference of the values of $u^{\star}(x)$ on $S_{i}^{R}$ and $S_{i}^{L}$ be $N / R$. Such function is evidently in $\mathcal{P}$.

We plug the function $u^{\star}(x)$ into expression (42). Since $\nabla u^{\star}(x)=0$ for all $x$ outside the tubes $S_{i}$ we have

$$
\frac{1}{N^{2}} \int_{\Lambda_{N}} \sigma(x)\left|\nabla u^{\star}(x)\right|^{2} \mathrm{~d} x=\frac{1}{N^{2}} \sum_{i=1}^{R} \int_{S_{i}} \sigma(x)\left|\nabla u^{\star}(x)\right|^{2} \mathrm{~d} x .
$$

The value of $\left|\nabla u^{\star}(x)\right|^{2}$ we can bounded from above by

$$
\left|\nabla u^{\star}(x)\right|^{2} \leqslant \frac{1}{4 d^{2}} \cdot \frac{N^{2}}{R^{2}}
$$

Indeed, let $x$ be an arbitrary point in $S_{i}$ and let $s_{x} \ni x$ be the segment perpendicular to $\tilde{S}_{i}$ with the length $2 d$ centred at $\tilde{S}_{i}$. The difference of the values of $u^{\star}$ on the ends of $s_{x}$ is by definition $N / R$ and function $u^{\star}$ is linear on $s_{x}$. Hence, the value of derivative of $u^{\star}$ in the direction of $s_{x}$ is $N / 2 d R$. It remains to check that the derivative of $u^{\star}(x)$ in the direction perpendicular to $s_{x}$ is zero. However, it is easy to verify using the fact that $\tilde{S}_{i}$ is composed by segments and pieces of circle, and that it is smooth.

We proceed by bounding the value of $\sigma(x)$. To achieve it, we divide every tube $S_{i}$ into two disjoint regions. The good one $\boldsymbol{S}_{i}^{g}=\boldsymbol{S}_{i} \cap\left\{x \in \mathbb{R}^{2}: S(x) \leqslant S_{c}(\lambda)+\varepsilon\right\}$ and the bad one $\boldsymbol{S}_{i}^{b}=\boldsymbol{S}_{i} \backslash \boldsymbol{S}_{i}^{g}$.

For $x \in S_{i}^{g}$, the conductivity $\sigma(x)$ is smaller than $\exp \left(\mu\left(S_{c}(\lambda)+\varepsilon\right)\right)$. To control the value of $\sigma(x)$ inside $\boldsymbol{S}_{i}^{b}$ we observe that $S_{i}^{b}$ consists of parts similar to the striped regions on Fig. 2. It is easy to check that there exists a constant $c_{1}>0$ such that for $d$ small enough the area of one such piece is smaller than $c_{1} d^{3}$. Similarly, we can find a constant $c_{2}>0$ such that the conductivity in the bad parts is bounded from above by $\exp \left(\mu\left(S_{c}(\lambda)+\varepsilon+c_{2} d^{2}\right)\right)$. 


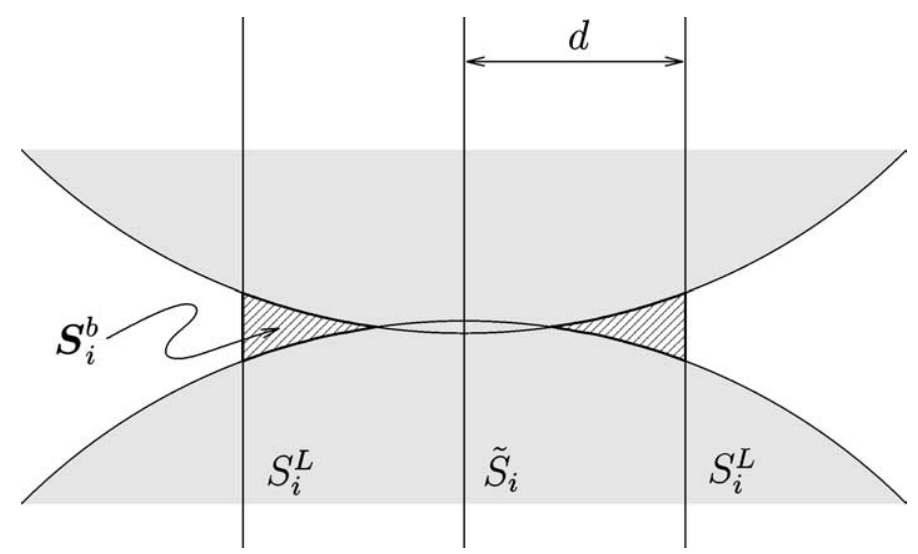

Fig. 2. Bad region of $S_{i}$.

Hence, we have

$$
\int_{S_{i}} \sigma(x)\left|\nabla u^{\star}(x)\right|^{2} \mathrm{~d} x=\int_{\boldsymbol{S}_{i}^{g}} \sigma(x)\left|\nabla u^{\star}(x)\right|^{2} \mathrm{~d} x+\int_{\boldsymbol{S}_{i}^{b}} \sigma(x)\left|\nabla u^{\star}(x)\right|^{2} \mathrm{~d} x
$$

with

$$
\int_{S_{i}^{g}} \sigma(x)\left|\nabla u^{\star}(x)\right|^{2} \mathrm{~d} x \leqslant \frac{1}{4 d^{2}} \cdot \frac{N^{2}}{R^{2}} \exp \left(\mu\left(S_{c}(\lambda)+\varepsilon\right)\right)\left|S_{i}\right|,
$$

and

$$
\int_{S_{i}^{b}} \sigma(x)\left|\nabla u^{\star}(x)\right|^{2} \mathrm{~d} x \leqslant \frac{1}{4 d^{2}} \cdot \frac{N^{2}}{R^{2}} \exp \left(\mu\left(S_{c}(\lambda)+\varepsilon+c_{2} d^{2}\right)\right) c_{1} d^{3} N_{b},
$$

where we use $N_{b}$ to denote the number of bad pieces and $|A|$ to denote the Lebesgue measure of the set $A \subset \mathbb{R}^{2}$.

Since we try to find the result on the logarithmic scale only, we can use a rather crude bound, $\left|\bigcup_{i} S_{i}\right| \leqslant N^{2}$. We also claim that there exists a constant $c_{3}$ depending only on $\lambda$ such that $N_{b} \leqslant c_{3} N^{2}$. The easiest way to see it, is to observe that bad pieces can come up only if there are two disks that almost touch in $\Lambda_{N}$. It is not possible to pack more than $\mathrm{O}\left(N^{2}\right)$ disks that almost touch on $R$ crossings of the box $\Lambda_{N}$. Putting all these estimates in expression (42) we get

$$
\begin{aligned}
\sigma^{\star}(\lambda, \mu) \leqslant & \lim _{N \rightarrow \infty}\left\{\frac{1}{N^{2}} \cdot \frac{1}{4 d^{2}} \cdot \frac{N^{2}}{R^{2}} \exp \left[\mu\left(S_{c}(\lambda)+\varepsilon\right)\right] N^{2}\right. \\
& \left.+\frac{1}{N^{2}} \cdot \frac{1}{4 d^{2}} \cdot \frac{N^{2}}{R^{2}} \exp \left[\mu\left(S_{c}(\lambda)+\varepsilon+c_{2} d^{2}\right)\right] c_{3} N^{2} c_{1} d^{3}\right\} .
\end{aligned}
$$

By Proposition 3.1(a) and Borel-Cantelli lemma for $\mathbb{P}$-a.e. realisation of the medium there is $N_{0}$ such that $R \geqslant \beta_{\varepsilon} N$ for all $N \geqslant N_{0}$. Hence, we have with probability one

$$
\sigma^{\star}(\lambda, \mu) \leqslant K d^{-2} \beta_{\varepsilon}^{-2} \mathrm{e}^{\mu\left(S_{c}(\lambda)+\varepsilon\right)}+K^{\prime} d \beta_{\varepsilon}^{-2} \mathrm{e}^{\mu\left(S_{c}(\lambda)+\varepsilon+c_{1} d^{2}\right)}=\mathrm{e}^{\mu\left(S_{c}(\lambda)+\varepsilon\right)} \beta_{\varepsilon}^{-2}\left(K \frac{1}{d^{2}}+K^{\prime} d \mathrm{e}^{\mu d^{2}}\right),
$$

where $K, K^{\prime}$ are the constants that do not depend on $\mu, d$ and $\varepsilon$. From the last expression we easily get

$$
\frac{1}{\mu} \log \sigma^{\star}(\lambda, \mu) \leqslant S_{c}(\lambda)+\varepsilon+d^{2}+\frac{1}{\mu}\left[2 \log \beta_{\varepsilon}-\log d+K^{\prime \prime}\right] .
$$


We now set $d=d(\mu)=\exp \left(-\mu^{1 / 2}\right)$ and compute the limit $\mu \rightarrow \infty$ of the last display. We obtain

$$
\limsup _{\mu \rightarrow \infty} \frac{1}{\mu} \log \sigma^{\star}(\lambda, \mu) \leqslant S_{c}(\lambda)+\varepsilon .
$$

Since $\varepsilon$ was arbitrary this gives the required upper bound.

Lower bound: For the lower bound we use the standard variational formula for the inverse of the homogenised matrix (see Chapters 1 and 8 of [7] for its proofs for periodic, resp. random setting). The isotropic version of such formula can be written as

$$
\left(\sigma^{\star}\right)^{-1}=\inf _{\boldsymbol{f} \in \mathcal{V}_{\text {sol }}^{2}} \frac{1}{N^{2}} \int_{\Lambda_{N}} \sigma(x)^{-1}\left(\boldsymbol{e}^{1}+\boldsymbol{f}(x)\right)^{2} \mathrm{~d} x,
$$

where $\mathcal{V}_{\text {sol }}^{2}=\left\{\boldsymbol{f}=\left(f_{1}, f_{2}\right): f_{1}, f_{2} \in L^{2}\left(\Lambda_{N}\right)\right.$, div $\left.\boldsymbol{f}=0, \int_{\Lambda_{N}} \boldsymbol{f}(x) \mathrm{d} x=0\right\}$, and $\boldsymbol{e}^{1}$ is the unit vector in $x$ direction.

Formula (57) can be rewritten using the fact that every function $\boldsymbol{f} \in \mathcal{V}_{\text {sol }}^{2}$ can be written as $\boldsymbol{f}=\left(\frac{\partial v}{\partial x^{2}},-\frac{\partial v}{\partial x^{1}}\right)$ for some function $v \in H^{1}\left(\Lambda_{N}\right)$ that satisfies $v \equiv 0$ on $\partial \Lambda_{N}$. Setting $u\left(x^{1}, x^{2}\right)=v\left(-x^{2}, x^{1}\right)+x^{1}$, we have $\nabla u(x)=$ $\boldsymbol{e}^{1}+\boldsymbol{f}(x)$. Thus (57) yields

$$
\frac{1}{\sigma^{\star}}=\lim _{N \rightarrow \infty} \frac{1}{N^{2}} \inf _{u \in \mathcal{P}^{\prime}} \int_{\Lambda_{N}} \sigma^{-1}(x)|\nabla u(x)|^{2} \mathrm{~d} x,
$$

where $\mathcal{P}^{\prime}=\left\{u \in H^{1}\left(\Lambda_{N}\right): u\left(x^{1}, x^{2}\right)=x^{1}\right.$ on $\left.\partial \Lambda_{N}\right\}$. This is the same variational formula as we used for the proof of the upper bound only with $\sigma$ replaced by $\sigma^{-1}$ and with $\mathcal{P}$ replaced by $\mathcal{P}^{\prime}$. The second change corresponds to the change of boundary conditions. Since the boundary conditions do not influence the value of the effective conductivity we replace $\mathcal{P}^{\prime}$ in (58) by $\mathcal{P}$. It allows us to use almost the same test-function as in the upper bound. The only difference is that the role of insulating grains and highly conducting domains between them will be reversed.

As in the proof of the upper bound we start by temporary rescaling of the box $\Lambda_{N}$. This time disks with radius $S_{c}(\lambda)-\varepsilon$ become disks with radius one. Using the same reasoning as in Eqs. (44) and (46) we find that the density $\lambda^{\prime}$ of the rescaled point process is smaller than $\lambda_{c}$. According to Proposition 3.1(b), there are at least $\beta^{\prime}\left(\lambda^{\prime}\right) N\left(2\left(S_{c}(\lambda)-\varepsilon\right)\right)^{-1} \equiv \beta_{\varepsilon}^{\prime} N$ vacant crossings of rescaled box. Returning to the original scale we obtain the same number of paths traversing $\Lambda_{N}$ in the complement of disks with radius $S_{c}(\lambda)-\varepsilon$.

We now use these crossings to construct the tubes similarly as in the upper bound. First note, that we can always deform them in the way that they will become once differentiable and will have the curvature bounded from above. We denote these smooth curves by $\tilde{S}_{i}, i=1, \ldots, R$, and we construct the tubes $\boldsymbol{S}_{i}$ with the sufficiently small radius $d$ and the function $u^{\star}(x)$ as before. The value of $\left|\nabla u^{\star}\right|^{2}$ in $S_{i}$ is bounded from above by

$$
\left|\nabla u^{\star}(x)\right|^{2} \leqslant \frac{1}{4 d^{2}} \cdot \frac{N^{2}}{R(\omega)^{2}}
$$

and is zero in the rest of $\Lambda_{N}$. For $\sigma^{-1}(x)$ the following bound is valid in $S_{i}$,

$$
\sigma^{-1}(x) \leqslant \exp \left[-\mu\left(S_{c}(\lambda)-\varepsilon-d\right)\right] .
$$

Plugging these two estimates into (58) we get

$$
\frac{1}{\sigma^{\star}} \leqslant \lim _{N \rightarrow \infty} \frac{1}{N^{2}} \exp \left[-\mu\left(S_{c}(\lambda)-\varepsilon-d\right)\right] \frac{1}{4 d^{2}} \cdot \frac{N^{2}}{R^{2}} \sum_{i=1}^{R}\left|\boldsymbol{S}_{i}\right| .
$$


We bound the last sum by $N^{2}$ and use the fact that with overwhelming probability $R \geqslant \beta_{\varepsilon}^{\prime} N$. Taking the logarithm we get

$$
\frac{1}{\mu} \log \sigma^{\star}(\lambda, \mu) \geqslant S_{c}(\lambda)-\varepsilon-d-\frac{1}{\mu}\left[2\left|\log \beta_{\varepsilon}^{\prime}\right|-\log d+K\right] .
$$

Setting $d=d(\mu)=\exp \left(-\mu^{1 / 2}\right)$ we obtain

$$
\liminf _{\mu \rightarrow \infty} \frac{1}{\mu} \log \sigma^{\star}(\lambda, \mu) \geqslant S_{c}(\lambda)-\varepsilon
$$

Since $\varepsilon$ was arbitrary it proves the lower bound.

Proof of Theorem 2.2. From the fact

$$
\hat{\sigma}_{\rho, \delta}(x, \mu) \leqslant \tilde{\sigma}_{\rho}(x, \mu) \leqslant \sigma(x, \mu)
$$

and the variational formula (42) we easily get the upper bound,

$$
\limsup _{\mu \rightarrow \infty} \frac{1}{\mu} \log \hat{\sigma}_{\rho, \delta}^{\star}(\lambda, \mu) \leqslant \limsup _{\mu \rightarrow \infty} \frac{1}{\mu} \log \tilde{\sigma}_{\delta}^{\star}(\lambda, \mu) \leqslant S_{c}(\lambda) .
$$

The dual variational formula (58) together with (64) imply that it is sufficient to prove the lower bound only for $\hat{\sigma}_{\rho, \delta}(x, \mu)$. We use the usual strategy to show it.

Let $\varepsilon>0$ such that $\varepsilon \leqslant \delta$. We rescale $\Lambda_{N}$ in such a way that the disks with radius $S_{c}(\lambda)-\varepsilon$ become the disks with radius one. As in the proof of the lower bound for Theorem 2.1 we receive the process with sub-critical density $\lambda^{\prime}$. The image of $\mathcal{V}_{\varepsilon}$ in this scaling is the set $\mathcal{W}$ defined in Proposition 3.7. As proved in that proposition there are at least $\beta^{\prime \prime}\left(\lambda^{\prime}\right) N\left(2\left(S_{c}(\lambda)-\varepsilon\right)\right)^{-1} \equiv \beta_{\varepsilon}^{\prime \prime} N$ crossings of the rescaled box in $\mathcal{W}$. If we return back to the original scale, we conclude that there is $\beta_{\varepsilon}^{\prime \prime} N$ crossings of $\Lambda_{N}$ in $\mathcal{V}_{\varepsilon}$. Moreover, it is not difficult to check that every crossing in $\mathcal{V}_{\varepsilon}$ can be smoothened in the way that the minimal radius of curvature is $\rho$ and the tubes with radius $\rho$ around the smooth version rest inside the tubes with the radius $\rho$ around $\mathcal{V}_{\varepsilon}$. We use $\tilde{S}_{i}$ to denote the smooth crossings. We choose $d<\rho$ and we construct the test-function $u^{\star}(x)$ in the same way as before. Since $S_{i} \subset\left\{x \in \mathbb{R}^{2}: d\left(x, \mathcal{V}_{\varepsilon}\right) \leqslant \rho\right\}$ and $\mathcal{V}_{\varepsilon} \subset \mathcal{V}_{\delta}$ we have

$$
\hat{\sigma}_{\rho, \delta}(x, \mu)=\sigma(x, \mu) \text { in } \boldsymbol{S}_{i} .
$$

After this observation the proof of the lower bound can be continued precisely in the same way as the proof of the lower bound for Theorem 2.1.

\section{References}

[1] V. Ambegaokar, B.I. Halperin, J.S. Langer, Hopping conductivity in disordered systems, Phys. Rev. B 4 (1971) 2612-2620.

[2] E. Charlaix, E. Guyon, S. Roux, Permeability of a random array of fractures of widely varying apertures, Transp. Porous Media 2 (1987) 31-43.

[3] M. Cieplak, A. Maritan, J.-R. Banavar, Optimal path and domain walls in the strong disorder limit, Phys. Rev. Lett. 72 (15) (1994) 2320-2323.

[4] K. Golden, S.M. Kozlov, Critical path analysis of transport in highly disordered random media, in: V. Berdichevsky, V. Jikov, G. Papanicolaou (Eds.), Homogenization, World Scientific, 1999, pp. 21-34.

[5] G. Grimmett, Percolation, second ed., Springer-Verlag, 1999.

[6] J.C. Gupta, B.V. Rao, Van den Berg-Kesten inequality for the Poisson boolean model for continuum percolation, Sankhya 61 (1999) 336-346.

[7] V.V. Jikov, S.M. Kozlov, O.A. Oleinik, Homogenization of Differential Operators and Integral Functionals, Springer-Verlag, 1994.

[8] A.J. Katz, A.H. Thompson, Quantitative prediction of permeability in porous rock, Phys. Rev. B 34 (11) (1986) 8179.

[9] T.M. Liggett, R.H. Schonmann, A.M. Stacey, Domination of product measures, Ann. Probab. 25 (1997) 71-95.

[10] R. Meester, R. Roy, Continuum Percolation, Cambridge University Press, 1996.

[11] C.M. Newman, D.L. Stein, Ground-state structure in a highly disordered spin-glass model, J. Statist. Phys. 82 (1996) $1113-1132$. 